\title{
BMJ Open Effectiveness of pharmacological and psychological interventions for treating post-traumatic stress disorder in adults with childhood abuse: protocol for a systematic review and network meta- analysis
}

Nour Waleed Alhussaini, Muhammad Riaz (1)

To cite: Alhussaini NW, Riaz M. Effectiveness of pharmacological and psychological interventions for treating post-traumatic stress disorder in adults with childhood abuse: protocol for a systematic review and network meta-analysis. BMJ Open 2021;11:e048790. doi:10.1136/ bmjopen-2021-048790

- Prepublication history for this paper is available online. To view these files, please visit the journal online (http://dx.doi. org/10.1136/bmjopen-2021048790).

Received 13 January 2021 Accepted 01 November 2021

Check for updates

(c) Author(s) (or their employer(s)) 2021. Re-use permitted under CC BY-NC. No commercial re-use. See rights and permissions. Published by BMJ.

Department of Public Health, College of Health Sciences, QU Health, Qatar University, Doha, Qatar

Correspondence to Dr Muhammad Riaz; medical.stn@gmail.com

\section{ABSTRACT}

Introduction Post-traumatic stress disorder (PTSD) is a debilitating mental disorder that develops after being exposed to a traumatic event. PTSD is common among adults who have experienced physical/sexual childhood abuse. Several psychological and pharmacological interventions are used for treating PTSD in this particular group, and it is important to identify what interventions, whether alone or in combination with other treatments, are more effective compared with others. Therefore, this review aims to provide synthesis of evidence on the effectiveness of different interventions used for treating PTSD following childhood abuse.

Methods and analysis Electronic search will be conducted using different databases such as PubMed, EMBASE, PsycINFO to identify randomised controlled trials (RCTs) used for assessing interventions for PTSD following childhood abuse. Data on treatment effectiveness for PTSD with childhood abuse and other variables will be extracted from each paper and reported as appropriate. Extracted effect-size estimates will be combined using Bayesian network meta-analysis (NMA). Risk of bias will be assessed through the Cochrane Collaboration tool for RCTs tool. NMA assumptions (heterogeneity, transitivity, inconsistency) will be assessed and reported. Metaregression and subgroup analyses will be performed to explore and explain possible sources of heterogeneity. Ethics and dissemination This research is based on literature review and does not require the approval of ethical board as it does not involve dealing with humans or animals. Findings of this review will be published in a peer-reviewed journal.

PROSPERO registration number CRD42020207409.

\section{BACKGROUND}

Being exposed to traumatic events is a part of human life and experience. A large number of studies indicate that it is common among humans to be exposed to traumatic events at some point during their lives. ${ }^{1-4}$ Findings of research showed that among the general
Strengths and limitations of this study

- This protocol is produced for conducting a comprehensive systematic review and network metaanalysis of published articles on the effectiveness pharmacological and psychological interventions used for treating post-traumatic stress disorder (PTSD) following childhood abuse.

- The results of this study will inform clinicians on the best choice of treatment for PTSD following childhood abuse.

- We have developed this protocol by following the Preferred Reporting Items for Systematic Reviews and Meta-Analyses.

- This protocol has been registered with International Prospective Register of Systematic Reviews.

- The study will rely on the existing evidence in the literature and may be subject to limitations in the published included studies.

population, a large number of people seem to be exposed to at least one traumatic event, with a prevalence ranging between $28 \%$ and $90 \% .^{5-12}$ There are different ways of responding to traumatic experiences as it can be identified in two ways. Some become resilient to their effects and can instantly recover, while others might develop symptoms that dissipate over time. Exposure to trauma is found to be associated with various types of physical as well as mental health issues. ${ }^{13-18}$ Although there is evidence that trauma is associated with different mental/ physical symptoms, how the occurrence of events affect individuals depends on certain factors, such as the definition of trauma, type of traumatic event exposed to, individual characteristics, sociocultural factors and developmental processes. ${ }^{19}$ There are various 
types of trauma: acute, chronic and complex. These types result from experiencing a single stressful event, repeated and prolonged stressful events, and exposure to multiple traumatic events, respectively. ${ }^{20}$ There are many different types of trauma and examples could be the unexpected death of a loved one, natural or human disasters, accidents, assaults during childhood or adulthood, including physical, mental or sexual abuse. ${ }^{21}$

The $\mathrm{WHO}^{22}$ defines child abuse as any form of 'physical and emotional mistreatment, sexual abuse, neglect, and negligent treatment of children, as well as to their commercial or other exploitation' (p7). Commercial or other exploitation refers to using children for the benefit of others, such as child labour and child prostitution, as well as using them in armed conflict and child trafficking. ${ }^{23}$ There are four main types of child abuse: physical, sexual abuse, emotional and psychological abuse. ${ }^{22} \mathrm{~A}$ global systematic review and meta-analysis showed that a minimum of over 1 billion children aged between 2 and 17 years old were exposed to any form of abuse (physical, emotional and sexual) in 2014. ${ }^{24}$ Even though there is evidence of the number of abused children worldwide, these numbers might be under-reported since they only include those who are reported to child protection agencies and organisations. Consequences of childhood maltreatment might not only be evident immediately after exposure to trauma, but also effects can be there for many years later. ${ }^{25}$

There are many different reasons why childhood abuse is more harmful to mental health than other types of traumas. For instance, this type of trauma occurs early in development, which might affect the course of personality development of the child as well as influence how they view themselves and the world, which might have long-lasting effects on their lives. Besides, this type of trauma seems to be chronic since it might be repeated or experienced for an extended time period. Finally, in some cases, when the perpetrators are parents or relatives who committed the abuse, they will have extreme betrayal feelings and will have issues with interpersonal functioning. ${ }^{25}$

Child abuse is considered as one type of trauma that results in many mental comorbidities, including posttraumatic stress disorder (PTSD).$^{26}$ PTSD is highly prevalent among those who have experienced childhood abuse, ${ }^{3}$ as it was reported in multiple studies that physically or sexually abused children have high rates of PTSD. ${ }^{27-30}$ The prevalence of women with PTSD who experienced childhood abuse and other related interpersonal violence ranged between $26 \%$ and $52 \% .{ }^{331} 32$ The results of a metaanalysis studying risk factors for PTSD presented findings of three main risk factors for the comorbidity, including history of childhood trauma. ${ }^{33}$ More specifically, those who experienced sexual abuse during childhood have a higher risk of developing this disorder compared with those who were not abused. ${ }^{34}$

People with PTSD might have disturbing feelings that could be related to the traumatic experience and that could last long after the event has ended. ${ }^{20}$ Symptoms of this disorder include re-experiencing the traumatic event in different distressing and vivid ways, like flashbacks, nightmares or frightening thoughts, avoiding places, objects, people, or even activities that remind them of the traumatic event, avoiding any thoughts related to the event, changes in cognition or mood (negative thoughts about the world and of oneself, distorted feelings, and detachment from others), and arousal and reactivity symptoms when reminded of the event (sleeping issues, reckless behaviours, poor concentration, anger outbursts). ${ }^{35} 36$ To be diagnosed with PTSD, one must have these symptoms for at least 1 month and facing distress and issues with daily functioning. ${ }^{35}$ PTSD patients might also face brain functional changes which includes fear responsiveness, emotion regulation, and deficits in declarative memory. ${ }^{37}$

There are a variety of pharmacological and psychological interventions for treating PTSD. ${ }^{38}$ Some evidence for the effectiveness of specific treatments for adults with PTSD following childhood abuse is available in the literature. ${ }^{39}$ However, we hypothesise that there will be differences in the effectiveness of treatments used for PTSD due childhood abused compared with other traumas. Therefore, the results of this review will allow us to compare the effectiveness of interventions among PTSD and other types of traumas. Pharmacological treatments are proposed to work by correcting imbalances in neurotransmitters that are responsible for causing PTSD and promoting structural brain functions. ${ }^{40} 41$ Two medications are approved to be used for treating PTSD, which are sertraline and paroxetine. ${ }^{42}$ Other pharmacological treatments used to improve PTSD symptoms include; selective serotonin reuptake inhibitors; serotonin and norepinephrine reuptake inhibitors; tricyclic antidepressants; benzodiazepines; mood stabilisers; atypical antipsychotics; adrenergic agents. ${ }^{43} 44$

Psychological treatments are also called talking therapies. These treatments help patients understand and cope with their own feelings. There are specific types of psychological treatments that were used for PTSD, which include trauma-focused cognitive behavioural therapy (CBT), eye movement desensitisation and reprocessing (EMDR), brief eclectic psychotherapy, interpersonal therapy, psychodynamic therapy and other therapies performed individually or in groups. These treatments aim to minimise the symptoms of PTSD, which are intrusion, avoidance and hyperarousal, through different ways of working through traumatic memories, changing patterns of thoughts and beliefs, managing stressors that are related to trauma, ${ }^{38}$ and improving functional brain regions that are responsible for extinction learning and re-appraisal. ${ }^{41}$

A variety of network meta-analyses (NMAs) related to PTSD were performed, covering different aspects, and filling certain gaps in the literature. For instance, some reviews focused on comparing the efficacy of psychological and medical treatments for PTSD in adults ${ }^{45} 46$ and 
among women with childhood abuse-related complex trauma. ${ }^{47}$ Another study compared treatments for patients with PTSD and other mental comorbidities following exposure to complex traumatic events. ${ }^{48}$ No available review focused on comparing the effectiveness of different types of treatments for individuals with PTSD after experiencing child abuse.

Because of the availability of various options for PTSD treatments in patients with childhood abuse, it is clinically important to identify what interventions, whether alone or in combination with other treatments, are found to be more effective compared with others. ${ }^{46}$ Furthermore, it is important to investigate what treatment is effective for subgroups of individuals with PTSD following childhood abuse. Hence, the main aim of this study is to provide a synthesis of evidence on the effectiveness of pharmacological and psychological interventions for treating PTSD in those who have experienced childhood abuse.

\section{Objectives}

The objectives of this review include:

- Provide overview of randomised controlled trials that have compared pharmacological and psychological interventions for PTSD in adults with childhood abuse.

- Quantitatively assess the clinical effectiveness of treatments delivered to PTSD patients who were victims of childhood abuse.

- Qualitatively assess the safety of treatments for people with PTSD following childhood abuse.

- Compare the effectiveness of pharmacological and psychological interventions through NMA.

- To assess the relative effectiveness of all available interventions by ranking their effect estimates from strongest to weakest using the Bayesian random effect model for NMA.

\section{METHODS}

This review was registered in PROSPERO. Preferred Reporting Items for Systematic Reviews and MetaAnalyses (PRISMA) extension statement for NMA and for reporting systematic reviews will be used throughout the research process. ${ }^{49}$ The Preferred Reporting Items for Systematic Review and Meta-Analysis Protocols checklist was used for reporting the current protocol. ${ }^{50}$

\section{Inclusion and exclusion criteria}

\section{Studies and setting}

We will include randomised controlled trials (RCT) and relevant cluster RCTs which have assessed any type of psychological or pharmacological intervention to treat patients with PTSD who were victims of childhood abuse. The RCTs could be blinded, double blinded or unblinded and may have two or more arms.

There will be no restriction on settings and place of the study as studies from across the globe with any setting will be included if they fulfil the inclusion criteria. To include a study in this review, articles need to be peer-reviewed original articles, measured the main outcome, assessed the effectiveness of the intervention and patients were victims of childhood abuse.

\section{Participants}

In the included studies, participants should be of any gender and aged 18 and above, diagnosed with PTSD and have experienced any of the four forms of childhood abuse (physical, sexual, emotional, neglect). Studies reporting different types of traumas in addition to child abuse will be considered and included in this review. Studies recruiting children or adolescents will be excluded. Studies reporting patients with comorbidities will be used to conduct sub-group analysis.

\section{Interventions}

Pharmacological (eg, sertraline or paroxetine or etc) and psychological (eg, CBT or EMDR or etc) interventions examined in RCTs for treating PTSD patients who had childhood abuse will be included in this study.

\section{Comparator}

This systematic review will be based on RCTs of psychological and pharmacological interventions. The psychological interventions are usually compared with a usual treatment, waiting list or any other alternative treatment. The pharmacological interventions are assessed against placebo, another treatment or psychotherapies.

\section{Outcome measure}

The main study outcome will be clinically significant reduction in the severity of PTSD symptoms in patients who were exposed to childhood abuse using standardised and validated scales.

A secondary outcome of safety of the intervention will also be considered which will be estimated from the reported withdrawals from the study due to adverse effects.

\section{Search methods}

\section{Electronic searches}

We will conduct the literature searches in bibliographic databases of PubMed, EMBASE, PsycINFO, Scopus, Web of Science, Published International Literature on Traumatic Stress, Cochrane Central Register and Google Scholar. The search strategy will be designed to capture all articles in English. However, if articles in other languages were found, all will be translated into English and included. All articles published until October 2021 and retrievable from electronic database will be considered for inclusion. The main search terms will be "posttraumatic stress disorder" AND "childhood abuse" AND "treatment". For example, the following search codes will be applied to all databases: ("posttraumatic stress disorder" OR PTSD OR stress* OR depress*) AND (child* abuse) AND (psycho* OR interventions OR treat* OR "trauma-focused cognitive behavioural therapy" OR CBT OR "group psychotherapies" OR "psychosocial functioning" OR "eye movement desensitization and reprocessing" OR EMDR OR "brief 
eclectic psychotherapy" OR "interpersonal therapy" OR "psychodynamic therapy" OR Sertraline OR Paroxetine OR Melitracen OR Amersergide OR Fluparoxan OR Demexiptiline OR Clomipramine OR Amitriptyline OR Lofepramine OR Fluoxetine OR Clovoxamine OR Deanol OR Amineptine OR Noxiptiline OR Mianserin OR Imipramine OR Diclofensine OR Teniloxine OR Rolipram OR Venlafaxine OR Idazoxan OR Butriptyline OR Fluotracen OR Tetrindole OR Oxaflozane OR Prosulpride OR Escitalopram OR Medifoxamine OR Caroxazone OR Alaproclate OR Brofaromine OR Oxaprotiline OR Zimeldine OR Opipramol OR Acetylcarnitine OR Pargyline OR Viloxazine OR Duloxetine OR Thiazesim OR Nialamide OR Femoxetine OR Milnacipran OR Pirlindole OR Tianeptine OR Befloxatone OR Amoxapine OR Viqualine OR Nefazodone OR Fluvoxamine OR Deprenyl OR Reboxetine OR Setiptiline OR Toloxatone OR Cilosamine OR Amiflamine OR Thozalinone OR Dibenzipin OR Benactyzine OR Doxepin OR Trazodone OR Phenelzine OR Nomifensine OR Protriptyline OR Citalopram OR Moclobemide OR Piribedil OR Maprotiline OR Dothiepin OR Bupropion OR Cimoxatone OR Quinupramine OR Desipramine OR Pivagabine OR Tranylcypromine OR Etoperidone OR Iprindole OR isocarboxazid OR Litoxetine OR Mirtazapine OR Tomoxetine OR Metapramine OR Trimipramine OR Iproniazid OR Minaprine)

\section{Manual search in reference lists}

In addition to the above-mentioned electronic search, we will conduct a manual search in references of included studies and already published systematic reviews related to our topic. All studies will be screened based on their titles and abstracts to examine their eligibility for inclusion.

\section{Selection of studies}

All studies obtained through the search strategy will be examined by at least two independent reviewers for eligibility criteria outlined above. After identifying eligible studies, all references will be added into a bibliographic referencing software EndnoteX $9^{51}$ to remove any duplicates. PRISMA flowchart will be used to record the inclusion and exclusion of studies. ${ }^{52} \mathrm{~A}$ table of the "Characteristics of included studies' will also be constructed.

\section{Data extraction}

Studies will be screened, and data will be extracted from all included studies. A predefined data extraction spreadsheet including main outcome of PTSD and its measure, types of childhood abuse, interventions assessed and comparator, sample/population, RCT type, study setting, country and statistical analysis, will be used. This will be further modified to accommodate further variables if required. At least two researchers will independently extract the data from all included studies. The extracted data will be compiled into appropriate tables and a narrative report will be presented.

\section{Quality assessment}

Quality assessment for included studies will be performed using the Cochrane Collaboration tool for RCTs to evaluate risk of bias (RoB).$^{53}$ The tool will be used to assess each study based on domains that reflect bias in RCTs. These domains are selection, detection, reporting, attrition, performance and other bias. A total score will be computed and final judgement of the RoB will be made for each included study based on each domain. A sensitivity analysis will be performed after identifying studies with high RoB and excluding them. Results from this sensitivity analysis will be reported as appropriate.

\section{Assessment of transitivity assumption}

A number of underlying assumptions must meet to perform an NMA. ${ }^{54}$ These assumptions include transitivity assumption. Transitivity refers to the validity of indirect comparisons of a network of treatments. Such comparisons might lead to confounding which results due to differences in trials. Transitivity can also refer to similarity in clinical and methodological characteristics. ${ }^{55}$ To meet the transitivity assumption, the distribution of effect modifiers should be similar across comparisons of interventions. Therefore, to meet this assumption, we will compare included studies if they are done in similar ways and recruited participants with an average of similar characteristics that might modify treatment effects. ${ }^{56}$

\section{Data synthesis and statistical analysis \\ Quantitative analysis}

A pairwise meta-analysis is commonly used to compare two interventions, whereas the NMA is used to compare several interventions simultaneously with combining direct and indirect treatments across a network of studies in one single analysis. Direct comparisons of treatments are obtained from studies that compare two treatments directly, while for the indirect comparisons, each treatment is used in a different trial as they were not compared directly. ${ }^{57}$

We anticipate that PTSD symptoms scores would have been used as the outcome measure in majority of studies, therefore, if an intervention is effective, a clinically relevant significant reduction in PTSD score could have occurred in the intervention group compared with the control. If PTSD symptoms scores have been used as a continuous outcome, we will extract the effect size as the standardised mean difference (Hedges's adjusted g). If this estimate is not available in a study, we will extract relevant statistics to compute it. If studies reported PTSD as a dichotomous outcome, we will extract/compute OR as an effect estimate. Our systematic review and NMA will only include RCTs, and we anticipate that majority of included studies will report outcome measure based on interviews rather self-reported questionnaire. Our emphasis will be using outcome measure from interviews. However, if both measures are available from enough number of studies, we will pool data on both measures.

We believe a considerable number of interventions from various studies and some pairs of the interventions would 
have not been directly compared in RCTs (eg, fluoxetine vs paroxetine or fluoxetine+CBT vs paroxetine). Furthermore, there could be multiple overlapping assessment in some of the studies (eg, fluoxetine vs paroxetine, fluoxetine vs sertraline or fluoxetine vs CBT), this may produce inconsistent estimates of the effects. Therefore, we aim to use NMA with Bayesian hierarchical random-effects mode ${ }^{58}$ to integrate the effect estimates and decide on an effective treatment among all available. This type of meta-analysis will allow us to estimate relative effects of two treatments with a proper probabilistic approach, as it can also allow us to estimate the ranking of efficacy for different interventions in an order. ${ }^{59}$

Heterogeneity among studies will be assessed by computing heterogeneity index Higgins $\mathrm{I}^{2}$ and by $\mathrm{Q}$ statistic. A comparison-adjusted funnel plots will be used to investigate for small study effects and for assessing publication bias. ${ }^{60}$ The R-package GeMTC ${ }^{61}$ (V.3.2.3), WinBUGS 1.4.3, ${ }^{62}$ or Stata V. $16^{63}$ will be used for the statistical analyses.

\section{Subgroup analysis}

A range of subgroup analyses will be performed to get insight about reasons for heterogeneity among studies. Some studies suggested that women have higher rates of PTSD compared with men ${ }^{64}$ therefore, subgroup analysis will be done on sex if sufficient studies are available. Other subgroups analyses will be conducted as follow if data was provided in included studies:

- Types of childhood abuse: whether physical, sexual or emotional abuse

- Study region (country)

- Single intervention versus combination: receive one treatment at a time or receive several treatments

- Psychological individual versus group interventions: receiving psychotherapy individually or in group of patients

- Acute, chronic, complex PTSD: number of traumatic events exposed to

- Severity of PTSD

- Type of intervention (pharmacological vs psychological)

- Type of psychological interventions

- Type of pharmacological interventions

\section{Consistency assessment}

Consistency is another assumption in NMA which refers to the agreement between direct and indirect estimates of the same treatment compared. ${ }^{545}$ This assumption can only be evaluated if a closed loop exists where there are direct and indirect comparisons of treatments. ${ }^{55}$ Consistency will be evaluated through statistically comparing direct and indirect summary effects.

\section{Qualitative analysis}

Qualitative analysis will be performed by examining safety of treatments and addressing all possible side effects in all included studies.

\section{Patient and public involvement \\ No patients involved.}

\section{ETHICS AND DISSEMINATION}

This research does not require the approval of ethical board as it does not involve dealing with humans or animals as it relies on reviewing of literature. Findings of this review will be published in a peer-reviewed journal.

Contributors The two authors (MR and NWA) have equally contributed to the design of this study and writing this protocol for publication.

Funding The authors have not declared a specific grant for this research from any funding agency in the public, commercial or not-for-profit sectors.

Competing interests None declared.

Patient and public involvement Patients and/or the public were not involved in the design, or conduct, or reporting, or dissemination plans of this research.

Patient consent for publication Not applicable.

Provenance and peer review Not commissioned; externally peer reviewed.

Open access This is an open access article distributed in accordance with the Creative Commons Attribution Non Commercial (CC BY-NC 4.0) license, which permits others to distribute, remix, adapt, build upon this work non-commercially, and license their derivative works on different terms, provided the original work is properly cited, appropriate credit is given, any changes made indicated, and the use is non-commercial. See: http://creativecommons.org/licenses/by-nc/4.0/.

ORCID iD

Muhammad Riaz http://orcid.org/0000-0002-5512-1745

\section{REFERENCES}

1 Breslau N, Kessler RC. The stressor criterion in DSM-IV posttraumatic stress disorder: an empirical investigation. Biol Psychiatry 2001;50:699-704.

2 Copeland WE, Keeler G, Angold A, et al. Traumatic events and posttraumatic stress in childhood. Arch Gen Psychiatry 2007;64:577-84.

3 Kessler RC, Sonnega A, Bromet E, et al. Posttraumatic stress disorder in the National comorbidity survey. Arch Gen Psychiatry 1995;52:1048-60.

4 McLaughlin KA, Koenen KC, Hill ED, et al. Trauma exposure and posttraumatic stress disorder in a national sample of adolescents. $J$ Am Acad Child Adolesc Psychiatry 2013;52:e14:815-30.

5 Norris FH. Epidemiology of trauma: frequency and impact of different potentially traumatic events on different demographic groups. $J$ Consult Clin Psychol 1992;60:409-18.

6 Breslau N, Kessler RC, Chilcoat HD, et al. Trauma and posttraumatic stress disorder in the community: the 1996 Detroit area survey of trauma. Arch Gen Psychiatry 1998;55:626-32.

7 Hepp U, Gamma A, Milos G, et al. Prevalence of exposure to potentially traumatic events and PTSD. The Zurich cohort study. Eur Arch Psychiatry Clin Neurosci 2006;256:151-8.

8 Storr CL, Schaeffer CM, Petras H, et al. Early childhood behavior trajectories and the likelihood of experiencing a traumatic event and PTSD by young adulthood. Soc Psychiatry Psychiatr Epidemiol 2009;44:398-406.

9 Roberts AL, Gilman SE, Breslau J, et al. Race/ethnic differences in exposure to traumatic events, development of post-traumatic stress disorder, and treatment-seeking for post-traumatic stress disorder in the United States. Psychol Med 2011;41:71-83.

10 Ogle CM, Rubin DC, Siegler IC. Cumulative exposure to traumatic events in older adults. Aging Ment Health 2014;18:316-25.

11 Knipscheer J, Sleijpen M, Frank L, et al. Prevalence of potentially traumatic events, other life events and subsequent reactions indicative for posttraumatic stress disorder in the Netherlands: a general population study based on the trauma screening questionnaire. Int J Environ Res Public Health 2020;17. doi:10.3390/ ijerph17051725. [Epub ahead of print: 0603 2020].

12 Kilpatrick DG, Resnick HS, Milanak ME, et al. National Estimates of Exposure to Traumatic Events and PTSD Prevalence Using DSM-IV and DSM-5 Criteria. J Trauma Stress 2013;26:537-47.

13 Turner RJ, Lloyd DA. Lifetime traumas and mental health: the significance of cumulative adversity. J Health Soc Behav 1995;36:360-76.

14 Norman SB, Means-Christensen AJ, Craske MG, et al. Associations between psychological trauma and physical illness in primary care. $J$ Trauma Stress 2006;19:461-70. 
15 Galea S, Brewin CR, Gruber M, et al. Exposure to hurricane-related stressors and mental illness after Hurricane Katrina. Arch Gen Psychiatry 2007;64:1427-34.

16 Spitzer C, Barnow S, Völzke H, et al. Trauma, posttraumatic stress disorder, and physical illness: findings from the general population. Psychosom Med 2009;71:1012-7.

17 Keyes KM, McLaughlin KA, Demmer RT, et al. Potentially traumatic events and the risk of six physical health conditions in a populationbased sample. Depress Anxiety 2013;30:451-60.

18 Scott KM, Koenen KC, Aguilar-Gaxiola S, et al. Associations between lifetime traumatic events and subsequent chronic physical conditions: a cross-national, cross-sectional study. PLoS One 2013;8:e80573.

19 Center for Substance Abuse Treatment (U.S.). Chapter 3Understanding the impact of trauma Trauma-Informed care in behavioral health services. Rockville, MD: U.S. Department of Health and Human Services, Substance Abuse and Mental Health Services Administration, Center for Substance Abuse Treatment, 2014.

20 Kira IA. Taxonomy of trauma and trauma assessment. Traumatology 2001;7:73-86.

21 Courtois CA, Sonis J, Brown LS. Clinical practice guideline for the treatment of posttraumatic stress disorder (PTSD) in adults. American Psychological Association, 2017.

22 Butchart A, Harvey AH. Preventing child maltreatment: a guide to taking action and generating evidence, 2006.

23 United Nations Children's Fund. Violence against children in East Asia and the Paci $\mathrm{C}$ a regional review and synthesis of findings. Bangkok, 2014

24 Hillis S, Mercy J, Amobi A, et al. Global prevalence of Past-year violence against children: a systematic review and minimum estimates. Pediatrics 2016;137:e20154079.

$25 \mathrm{Han} \mathrm{H.} \mathrm{A} \mathrm{longitudinal} \mathrm{assessment} \mathrm{of} \mathrm{cognitive} \mathrm{schemas} \mathrm{in} \mathrm{women}$ with posttraumatic stress disorder related to childhood abuse. New York: Fordham University, 2004.

26 Powers A, Etkin A, Gyurak A, et al. Associations between childhood abuse, posttraumatic stress disorder, and implicit emotion regulation deficits: evidence from a low-income, inner-city population. Psychiatry 2015;78:251-64.

27 Adam BS, Everett BL, O'Neal E. Ptsd in physically and sexually abused psychiatrically hospitalized children. Child Psychiatry Hum Dev 1992;23:3-8.

28 Famularo R, Kinscherff R, Fenton T. Psychiatric diagnoses of maltreated children: preliminary findings. J Am Acad Child Adolesc Psychiatry 1992;31:863-7.

29 Kinzie JD, Sack W, Angell R, et al. A three-year follow-up of Cambodian young people traumatized as children. J Am Acad Child Adolesc Psychiatry 1989;28:501-4.

30 Merry SN, Andrews LK. Psychiatric status of sexually abused children 12 months after disclosure of abuse. J Am Acad Child Adolesc Psychiatry 1994;33:939-44.

31 Kessler RC. Posttraumatic stress disorder: the burden to the individual and to Society. J Clin Psychiatry 2000;61 Suppl 5:4-12. discussion 3-4.

32 Ullman SE, Brecklin LR. Sexual assault history and suicidal behavior in a national sample of women. Suicide Life Threat Behav 2002;32:117-30.

33 Brewin CR, Andrews B, Valentine JD. Meta-Analysis of risk factors for posttraumatic stress disorder in trauma-exposed adults. $J$ Consult Clin Psychol 2000;68:748-66.

34 Rodriguez N, Ryan SW, Vande Kemp H, et al. Posttraumatic stress disorder in adult female survivors of childhood sexual abuse: a comparison study. J Consult Clin Psychol 1997;65:53-9.

35 Brewin CR, Cloitre M, Hyland P, et al. A review of current evidence regarding the ICD-11 proposals for diagnosing PTSD and complex PTSD. Clin Psychol Rev 2017;58:1-15.

36 The National Institute of Mental Health. Post-Traumatic stress disorder, 2019. Available: https://www.nimh.nih.gov/health/topics/ post-traumatic-stress-disorder-ptsd/index.shtml

37 Elzinga BM, Bremner JD. Are the neural substrates of memory the final common pathway in posttraumatic stress disorder (PTSD)? J Affect Disord 2002;70:1-17.

38 Jonas DE, Cusack K, Forneris CA, et al. Psychological and pharmacological treatments for adults with posttraumatic stress disorder (PTSD). AHRQ comparative effectiveness reviews. Rockville (MD: Agency for Healthcare Research and Quality, 2013.

39 Ehring T, Welboren R, Morina N, et al. Meta-Analysis of psychological treatments for posttraumatic stress disorder in adult survivors of childhood abuse. Clin Psychol Rev 2014;34:645-57.
40 Stein DJ, Seedat S, van der Linden GJ, et al. Selective serotonin reuptake inhibitors in the treatment of post-traumatic stress disorder: a meta-analysis of randomized controlled trials. Int Clin Psychopharmacol 2000;15 Suppl 2:S31-9.

41 Thomaes K, Dorrepaal E, Draijer N, et al. Can pharmacological and psychological treatment change brain structure and function in PTSD? A systematic review. J Psychiatr Res 2014:50:1-15.

42 U.S. Food and Drug Administration. Paxil NDA 20-031/S-029 approval letter, 2001.

43 Gutner CA, Casement MD, Stavitsky Gilbert K, et al. Change in sleep symptoms across cognitive processing therapy and prolonged exposure: a longitudinal perspective. Behav Res Ther 2013:51:817-22.

44 Hagl M, Powell S, Rosner R, et al. Dialogical exposure with traumatically bereaved Bosnian women: findings from a controlled trial. Clin Psychol Psychother 2015;22:604-18.

45 Cipriani A, Williams T, Nikolakopoulou A, et al. Comparative efficacy and acceptability of pharmacological treatments for post-traumatic stress disorder in adults: a network meta-analysis. Psychol Med 2018;48:1975-84.

46 Watts BV, Schnurr PP, Mayo L, et al. Meta-Analysis of the efficacy of treatments for posttraumatic stress disorder. J Clin Psychiatry 2013;74:e541-50.

47 Dorrepaal E, Thomaes K, Hoogendoorn AW, et al. Evidence-Based treatment for adult women with child abuse-related complex PTSD: a quantitative review. Eur J Psychotraumatol 2014;5:23613.

48 Coventry PA, Meader N, Melton H, et al. Psychological and pharmacological interventions for posttraumatic stress disorder and comorbid mental health problems following complex traumatic events: systematic review and component network meta-analysis. PLoS Med 2020;17:e1003262.

49 Hutton B, Salanti G, Caldwell DM, et al. The PRISMA extension statement for reporting of systematic reviews incorporating network meta-analyses of health care interventions: checklist and explanations. Ann Intern Med 2015;162:777-84.

50 Moher D, Shamseer L, Clarke M, et al. Preferred reporting items for systematic review and meta-analysis protocols (PRISMA-P) 2015 statement. Syst Rev 2015;4:1.

51 The EndNote Team. Endnote. EndNote X9 ed. Philadelphia, PA: Clarivate, 2013

52 Moher D, Liberati A, Tetzlaff J, et al. Preferred reporting items for systematic reviews and meta-analyses: the PRISMA statement. $J$ Clin Epidemiol 2009;62:1006-12.

53 Higgins JPT, Altman DG, Gotzsche PC, et al. The Cochrane Collaboration's tool for assessing risk of bias in randomised trials. BMJ 2011;343:d5928

54 Salanti G. Indirect and mixed-treatment comparison, network, or multiple-treatments meta-analysis: many names, many benefits, many concerns for the next generation evidence synthesis tool. Res Synth Methods 2012;3:80-97.

55 Cipriani A, Higgins JPT, Geddes JR, et al. Conceptual and technical challenges in network meta-analysis. Ann Intern Med 2013;159:130-7.

56 Airways Cochrane. The transitivity assumption. Available: https:// airways.cochrane.org/sites/airways.cochrane.org/files/public/ uploads/The\%20transitivity\%20assumption\%20CJC.pdf

57 Hu D, O'Connor AM, Wang C, et al. How to conduct a Bayesian network meta-analysis. Frontiers in Veterinary Science 2020;7.

$58 \mathrm{Lu} \mathrm{G}$, Ades AE. Combination of direct and indirect evidence in mixed treatment comparisons. Stat Med 2004;23:3105-24.

59 Chaimani A, Caldwell DM, Higgins JPT, et al. Chapter 11: undertaking network meta-analyses. Cochrane Handbook for systematic reviews of interventions, 2020.

60 Chaimani A, Salanti G. Using network meta-analysis to evaluate the existence of small-study effects in a network of interventions. Res Synth Methods 2012;3:161-76.

61 Valkenhoef GV, Kuiper J. Network meta-analysis using Bayesian methods. 0.8-7 ED, 2020.

62 Spiegelhalter D, Thomas A, WinBUGS BN. 1.4.3 ED. Cambridge, England: MRC Biostatistics Unit, Institute of Public Health, 2003.

63 StataCorp. Stata statistical software: release 16. College Station, TX: StataCorp LLC, 2019.

64 Hatch SL, Dohrenwend BP. Distribution of traumatic and other stressful life events by race/ethnicity, gender, Ses and age: a review of the research. Am J Community Psychol 2007;40:313-32.

65 Higgins JPT, Jackson D, Barrett JK, et al. Consistency and inconsistency in network meta-analysis: concepts and models for multi-arm studies. Res Synth Methods 2012;3:98-110. 